\title{
SEMI-CONTINUITY OF THE DIEDERICH-FORNÆSS AND STEINNESS INDICES
}

\author{
YOUNG-JUN CHOI AND JIHUN YUM
}

\begin{abstract}
In this paper, we prove the semi-continuity theorem of DiederichFornæss index and Steinness index under a smooth deformation of pseudoconvex domains in Stein manifolds.
\end{abstract}

\section{Introduction}

Let $\Omega$ be a relatively compact, Levi pseudoconvex domain in a complex manifold $X$ with $C^{\infty}$-smooth boundary. Let $\rho: X \rightarrow \mathbb{R}$ be a smooth defining function of $\Omega$, i.e., $\Omega=\{z \in X: \rho(z)<0\}$ and $d \rho \neq 0$ on $\partial \Omega$. The Diederich-Fornæss index $D F(\Omega)$ and the Steinness index $S(\Omega)$ of $\Omega$ are defined by

$$
\begin{aligned}
D F(\Omega) & :=\sup _{\rho}\left\{0<\gamma<1:-(-\rho)^{\gamma} \text { is strictly plurisubharmonic on } \Omega \cap W\right\}, \\
S(\Omega) & :=\inf _{\rho}\left\{\gamma>1: \rho^{\gamma} \text { is strictly plurisubharmonic on } \bar{\Omega}^{\complement} \cap W\right\},
\end{aligned}
$$

where the supremum and infimum are taken over all smooth defining function $\rho$, and $W$ is some neighborhood of $\partial \Omega$ that may depend on $\rho$ and $\gamma$. If such $\rho$ and $\gamma$ do not exist, we define $D F(\Omega)=0$ and $S(\Omega)=\infty$. When the ambient space $X$ is a Stein manifold, $D F(\Omega)>0$ implies the existence of a bounded strictly plurisubharmonic function on $\Omega$, i.e., $\Omega$ is hyperconvex, and $S(\Omega)<\infty$ implies that $\Omega$ admits a Stein neighborhood basis. In 1977, Diederich and Fornæss (5]) proved the positivity of $D F(\Omega)$ if the boundary is of $C^{2}$-smoothness. The second named author $([10])$ provided a necessary and sufficient condition for $S(\Omega)<\infty$.

In this paper, we shall study the semi-continuity of the both indices of a relatively compact pseudoconvex domain with smooth boundary under a smooth deformation. More precisely, a smooth deformation of a relatively compact domain $\Omega_{0}$ in a complex manifold $X_{0}$ is given as follows:

A surjective holomorphic submersion $\pi:(X, \Omega) \rightarrow \mathbb{D}$ from a complex manifold $X$ with a relatively compact domain $\Omega$ in $X$ to the unit disc $\mathbb{D}$ is called a smooth deformation of $\Omega_{0}$ in $X_{0}$ over $\mathbb{D}$ if

- $X_{0}=\pi^{-1}(0)$ and $\Omega_{0}=\Omega \cap X_{0}$.

- $\Omega$ admits a defining function $\delta$ such that $\left.\delta\right|_{X_{t}}$ is a defining function of $\Omega_{t}:=$ $\Omega \cap \pi^{-1}(t)$ in $X_{t}:=\pi^{-1}(t)$ for $t \in \mathbb{D}$.

Note that the above conditions guarantee that every fiber $\Omega_{t}$ is diffeomorphic to $\Omega_{0}$. (cf, see [9].)

The main theorem of this paper is as follows:

Date: April 14, 2020.

2010 Mathematics Subject Classification. 32T27, 32U10, 32G05.

Key words and phrases. Diederich-Fornæss index, Steinness index, deformation of pseudoconex domains. 
Theorem 1.1. If every fiber $X_{t}$ is a Stein manifold, then

$$
\liminf _{t \rightarrow 0} D F\left(\Omega_{t}\right) \geq D F\left(\Omega_{0}\right) \quad \text { and } \quad \limsup _{t \rightarrow 0} S\left(\Omega_{t}\right) \leq S\left(\Omega_{0}\right) .
$$

In other words, $D F\left(\Omega_{t}\right)$ is lower semi-continuous and $S\left(\Omega_{t}\right)$ is upper semi-continuous at $t=0$, respectively.

We would like to emphasize that the Steinness of each fiber $X_{t}$ is a superfluous condition. In fact, the condition in Theorem 2.3, which is our main tool, is sufficient. That is, if each $\Omega_{t}$ admits a defining function $\rho_{t}$ such that either $\bar{\partial} \omega_{\rho_{t}}>0$ on $\mathcal{N}$ or $\bar{\partial} \omega_{\rho_{t}}<0$ on $\mathcal{N}$, then we have the same conclusion. Here, $\omega_{\rho_{t}}$ is a D'Angelo $(1,0)$-form and $\mathcal{N}$ is the kernel of the Levi form (see Section 2 ). We refer readers to Corollary 5.4 and Remark 5.6 in [1] for the details.

Acknowledgment. The authors would like to thank A. Seo for suggesting the problem and M. Adachi for a useful comment on Lemma 3.1. This work was supported by the National Research Foundation (NRF) of Korea grant funded by the Korea government (No. 2018R1C1B3005963).

\section{Preliminaries}

In this section, we introduce the D'Angelo 1-form and the characterizations of Diederich-Fornæss and Steinness indices by the D'Angelo 1-form due to Adachi and the second named author ([1]).

First, we recall the definition of D'Angelo 1-form, which was introduced by D'Angelo ([3], 44]), and developed by Boas and Straube ([2]). Let $\Omega$ be a relatively compact, Levi pseudoconvex domain in a complex manifold $X$ with $C^{\infty}$-smooth boundary. Let $\rho$ be a smooth defining function of $\Omega$. Denote the kernel of the Levi form by $\mathcal{N}=\bigcup_{p \in M} \mathcal{N}_{p} \subset T^{1,0}(\partial \Omega)$ where

$$
\mathcal{N}_{p}:=\left\{L_{p} \in T_{p}^{1,0}(\partial \Omega) \mid \mathscr{L}_{\rho}\left(L_{p}, L_{p}^{\prime}\right)=0 \quad \forall L_{p}^{\prime} \in T_{p}^{1,0}(\partial \Omega)\right\}
$$

for each $p \in \partial \Omega$, where $\mathscr{L}_{\rho}$ is the Levi-form of $\rho$. Note that the kernel $\mathcal{N}$ does not depend on a defining function $\rho$, and $\mathcal{N}_{p}=\left\{L_{p} \in T_{p}^{1,0}(\partial \Omega) \mid \mathscr{L}_{\rho}\left(L_{p}, L_{p}\right)=0\right\}$ when $\Omega$ is a Levi pseudoconvex domain. Define

$$
\eta_{\rho}:=\frac{1}{2}(\partial \rho-\bar{\partial} \rho)
$$

which is a purely imaginary, non-vanishing 1 -form on $\partial \Omega$ that annihilates $T^{1,0}(\partial \Omega) \oplus$ $T^{0,1}(\partial \Omega)$. Let $T_{\rho} \in \Gamma(\mathbb{C} \otimes T(\partial \Omega))$ be a purely imaginary, non-vanishing, smooth vector field on $\partial \Omega$ such that $\eta_{\rho}\left(T_{\rho}\right)=1$. Then $T_{\rho}$ yields a decomposition

$$
\mathbb{C} \otimes T(\partial \Omega)=T^{1,0}(\partial \Omega) \oplus T^{0,1}(\partial \Omega) \oplus \mathbb{C} T_{\rho} .
$$

We call such $T_{\rho}$ a transversal vector field normalized with respect to $\eta_{\rho}$. Denote $\eta_{\rho}$ and $T_{\rho}$ by $\eta$ and $T$, respectively, if there is no ambiguity.

Definition 2.1. A D'Angelo 1-form $\alpha_{\rho}$ of $\rho$ on $\partial \Omega$ is defined by

$$
\alpha_{\rho}:=-\mathcal{L}_{T_{\rho}} \eta_{\rho}
$$

where $\mathcal{L}_{T_{\rho}}$ is the Lie derivative in the direction of $T_{\rho}$. A D'Angelo $(1,0)$-form $\omega_{\rho}:=$ $\pi_{1,0} \alpha_{\rho}$ is the projection of $\alpha_{\rho}$ onto its $(1,0)$-component. 
Remark 2.2. In [1], they defined the D'Angelo 1-form on a compact abstract CR manifold $M$ of hypersurface type without using a defining function. However, this definition is equivalent to Definition 2.1 when $M$ bounds a relatively compact domain in a complex manifold. In this paper, we use Definition 2.1 because we deal with only domains.

Note that although, for a defining function $\rho$, a transversal vector field $T_{\rho}$ normalized with respect to $\eta_{\rho}$ is not unique, $\omega_{\rho}$ and $\bar{\partial} \omega_{\rho}$ are well-defined on $\mathcal{N}$, that is, they are independent of the choice of $T_{\rho}$ (see Lemma 2.5 and 2.6 in [1]). We regard $\bar{\partial} \omega_{\rho}$ and $\omega_{\rho} \wedge \bar{\omega}_{\rho}$ as quadratic forms on $\mathcal{N}$, i.e., $\bar{\partial} \omega_{\rho}>0$ on $\mathcal{N}$ means $\bar{\partial} \omega_{\rho}(L, \bar{L})>0$ for all $L \in \mathcal{N}$. The main tool we will use in Section 3 is the following.

Theorem 2.3 (Adachi, Yum [1]). Suppose that there exists a defining function $\rho_{1}$ of $\Omega$ such that $\bar{\partial} \omega_{\rho_{1}}>0$ on $\mathcal{N}$ or $\bar{\partial} \omega_{\rho_{1}}<0$ on $\mathcal{N}$. Then

$$
\begin{aligned}
D F(\Omega) & =\sup _{\rho}\left\{0<\gamma<1: \bar{\partial} \omega_{\rho}-\frac{\gamma}{1-\gamma}\left(\omega_{\rho} \wedge \bar{\omega}_{\rho}\right)>0 \quad \text { on } \mathcal{N}\right\}, \\
S(\Omega) & =\inf _{\rho}\left\{\gamma>1:-\bar{\partial} \omega_{\rho}-\frac{\gamma}{\gamma-1}\left(\omega_{\rho} \wedge \bar{\omega}_{\rho}\right)>0 \quad \text { on } \mathcal{N}\right\} .
\end{aligned}
$$

If the supremum or infimum does not exist, then $D F(\Omega)=0$ or $S(\Omega)=\infty$, respectively.

\section{Semi-continuity of two indices}

In this section, we prove the main theorem. Let $\pi:(X, \Omega) \rightarrow \mathbb{D}$ be a smooth deformation of $\Omega_{0}$ in $X_{0}$ over $\mathbb{D}$. Let $\operatorname{dim}_{\mathbb{C}} X=n+1$ and $\operatorname{dim}_{\mathbb{C}} X_{t}=n$. Let $\delta$ be a defining function of $\Omega \subset X$ such that $\delta_{t}:=\left.\delta\right|_{X_{t}}$ is also a defining function of $\Omega_{t} \subset X_{t}$ for each $t \in \mathbb{D}$, and $M:=\partial \Omega$. For a point $p \in \partial \Omega_{t} \subset M$, denote by

$$
\mathcal{N}_{p}:=\left\{L_{p} \in T_{p}^{1,0}\left(\partial \Omega_{t}\right) \mid \mathscr{L}_{\delta_{t}}\left(L_{p}, L_{p}^{\prime}\right)=0 \quad \forall L_{p}^{\prime} \in T_{p}^{1,0}\left(\partial \Omega_{t}\right)\right\} .
$$

Lemma 3.1. For $p \in \partial \Omega_{0} \subset M$, suppose that $\operatorname{dim}_{\mathbb{C}}\left(\mathcal{N}_{p}\right)=m(1 \leq m \leq n-1)$. Then there exist a neighborhood $U_{p}$ of $p$ in $M$ and $m$ linearly independent smooth $(1,0)$ vector fields $L_{1}, \cdots, L_{m}$ on $U_{p}$ satisfying the following conditions:

- $\mathcal{N}_{p}=\left\langle L_{1}(p), \cdots, L_{m}(p)\right\rangle$,

- For each $q \in U_{p}, \mathcal{N}_{q} \subset\left\langle L_{1}(q), \cdots, L_{m}(q)\right\rangle$.

Proof. Choose a basis $\left\{L_{1, p}, \cdots, L_{n-1, p}\right\}$ for $T_{p}^{1,0}\left(\partial \Omega_{0}\right)$ such that $\left\{L_{1, p}, \cdots, L_{m, p}\right\}$ forms a basis for $\mathcal{N}_{p}$. Then, for each $j=1, \cdots, n-1$, we extend $L_{j, p}$ smoothly to a $(1,0)$ vector field $X_{j}$ on a neighborhood $U_{p}$ of $p$ in $M$ such that $X_{j}(p)=L_{j, p}$ and $\left\{X_{1}(q), \cdots, X_{n-1}(q)\right\}$ forms a basis for $T_{q}^{1,0}\left(\partial \Omega_{t}\right)$ for $q \in U_{p}$ with $\pi(q)=t$. This is possible because $\bigcup_{q \in \partial \Omega} T_{q}^{1,0}\left(\partial \Omega_{t}\right)$ is a vector subbundle of $T^{1,0}(\partial \Omega)$. Let $M(q)$ be the matrix representation of the Levi-form with respect to a basis $\left\{X_{j}(q)\right\}_{j=1}^{n-1}$ at $q \in U_{p}$, i.e., $M(q)=\left[M_{i j}(q)\right]_{(n-1) \times(n-1)}:=\left[\mathscr{L}_{\delta_{t}}\left(X_{i}(q), X_{j}(q)\right)\right]$. Then $M(q)$ is a hermitian matrix and let

$$
M(q)=\left[\begin{array}{ll}
A(q) & B(q) \\
B(q)^{*} & C(q)
\end{array}\right]
$$

with blocks of size $m$ and $(n-m-1)$, where $B(q)^{*}$ is the conjugate transpose of $B(q)$. Also, by the construction, $A(p)=B(p)=0$ and $C(p)$ is invertible. By the 
continuity, $C(q)$ is still invertible on $U_{p}$ (by shrinking $U_{p}$ if necessary). Let

$$
\Psi(q):=\left[\begin{array}{cc}
I_{m \times m} & 0 \\
-C(q)^{-1} B(q)^{*} & C(q)^{-1}
\end{array}\right],
$$

where $I_{m \times m}$ is the identity matrix. Define vector fields $L_{j}(q)(1 \leq j \leq n-1)$ on $U_{p}$ by

$$
\left[L_{1}(q) \cdots L_{n-1}(q)\right]:=\left[X_{1}(q) \cdots X_{n-1}(q)\right] \times \bar{\Psi}(q),
$$

where $\times$ is the matrix multiplication. Then $\mathcal{N}_{p}=\left\langle L_{1}(p), \cdots, L_{m}(p)\right\rangle$ because $L_{j}(p)=X_{j}(p)=L_{j, p}$ for $j=1, \cdots, m$, and the matrix representation of the Leviform with respect to a basis $\left\{L_{j}(q)\right\}_{j=1}^{n-1}$ is

$$
\Psi(q)^{*} \times M(q) \times \Psi(q)=\left[\begin{array}{cc}
A(q)-B(q) C(q)^{-1} B(q)^{*} & 0 \\
0 & C(q)^{-1}
\end{array}\right] .
$$

Therefore, since $C(q)^{-1}$ is invertible, we conclude that $\mathcal{N}_{q} \subset\left\langle L_{1}(q), \cdots, L_{m}(q)\right\rangle$.

Proof of Theorem 1.1. Since each fiber $X_{t}$ is a Stein manifold, one may apply Theorem 2.3 for each $\Omega_{t}$ (see Corollary 5.4 in [1]). By Theorem [2.3, for each $0<\gamma<$ $D F\left(\Omega_{0}\right)$, there exists a defining function $\rho: X_{0} \rightarrow \mathbb{R}$ of $\Omega_{0} \subset X_{0}$ such that

$$
\bar{\partial} \omega_{\rho}-\frac{\gamma}{1-\gamma}\left(\omega_{\rho} \wedge \bar{\omega}_{\rho}\right)>0 \quad \text { on } \mathcal{N}
$$

where $\mathcal{N}:=\bigcup_{p \in \partial \Omega_{0}} \mathcal{N}_{p} \subset T^{1,0}\left(\partial \Omega_{0}\right)$. We will extend $\rho$ smoothly to $\widetilde{\rho}: \pi^{-1}\left(U_{0}\right) \rightarrow \mathbb{R}$ such that $\widetilde{\rho}_{t}:=\left.\widetilde{\rho}\right|_{X_{t}}$ is a defining function of $\Omega_{t}$ for each $t \in U_{0}$ and $\widetilde{\rho}_{0}=\rho$, where $U_{0}$ is a neighborhood of $0 \in \mathbb{D}$. Since $\delta_{0}$ is also a defining function of $\Omega_{0} \subset X_{0}$, there exists a smooth function $\psi \in C^{\infty}\left(X_{0}\right)$ such that $\rho=e^{\psi} \delta_{0}$. Then since $X_{0}$ is a closed submanifold of $X$, we may extend $\psi$ to $\widetilde{\psi} \in C^{\infty}\left(\pi^{-1}\left(U_{0}\right)\right.$ ) (by shrinking $U_{0}$ if necessary), and $\widetilde{\rho}:=e^{\widetilde{\psi}} \delta$ is the desired function. We denote $\widetilde{\rho}$ by $\rho$ again, and $\omega_{\rho_{t}}$ by $\omega_{t}$.

Now for $p \in \partial \Omega_{0}$, suppose that $\operatorname{dim}_{\mathbb{C}}\left(\mathcal{N}_{p}\right)=m>0$. Then by Lemma 3.1, there exist a neighborhood $U_{p}$ of $p$ in $M$ and $m$ linearly independent smooth $(1,0)$ vector fields $L_{1}, \cdots, L_{m}$ on $U_{p}$ satisfying the following conditions:

- $\mathcal{N}_{p}=\left\langle L_{1}(p), \cdots, L_{m}(p)\right\rangle$,

- For each $q \in U_{p}, \mathcal{N}_{q} \subset\left\langle L_{1}(q), \cdots, L_{m}(q)\right\rangle$.

Then, by the continuity, the inequality (3.1) implies that there exists a neighborhood $V_{p} \subset U_{p}$ such that

$$
\bar{\partial} \omega_{t}-\frac{\gamma}{1-\gamma}\left(\omega_{t} \wedge \bar{\omega}_{t}\right)>0 \quad \text { on }\left\langle L_{1}(q), \cdots, L_{m}(q)\right\rangle
$$

for all $q \in V_{p}$ with $\pi(q)=t$. Since $\mathcal{N}_{q} \subset\left\langle L_{1}(q), \cdots, L_{m}(q)\right\rangle$,

$$
\bar{\partial} \omega_{t}-\frac{\gamma}{1-\gamma}\left(\omega_{t} \wedge \bar{\omega}_{t}\right)>0 \quad \text { on } \mathcal{N}_{q}
$$

for all $q \in V_{p}$ with $\pi(q)=t$. Therefore, since the set $\left\{p \in \partial \Omega_{0}: \operatorname{dim}_{\mathbb{C}}\left(\mathcal{N}_{p}\right)>0\right\}$ is compact, we conclude that

$$
\liminf _{t \rightarrow 0} D F\left(\Omega_{t}\right) \geq D F\left(\Omega_{0}\right)
$$

by applying Theorem 2.3 again. $\lim _{\sup _{t \rightarrow 0}} S\left(\Omega_{t}\right) \leq S\left(\Omega_{0}\right)$ follows from the same argument as above. 


\section{Example}

In 1977, Diederich and Fornæss ([6]) constructed a 1-parameter family of bounded, pseudoconvex domains $\Omega_{\beta}\left(\beta>\frac{\pi}{2}\right)$, called worm domains, in $\mathbb{C}^{2}$ with $C^{\infty}$-smooth boundaries. They showed that the Diederich-Fornæss indices of worm domains are non-trivial, i.e., $0<D F\left(\Omega_{\beta}\right)<1$, for all $\beta$, and $\Omega_{\beta}$ does not admit a Stein neighborhood basis for some $\beta$. Recently, Liu ([8]) calculated the exact value of the $D F\left(\Omega_{\beta}\right)$. Also, the second named author ([10]) calculated the exact value of the $S\left(\Omega_{\beta}\right)$ and found the following relation between two indices for worm domains:

$$
\frac{1}{D F\left(\Omega_{\beta}\right)}+\frac{1}{S\left(\Omega_{\beta}\right)}=2
$$

whenever $\operatorname{DF}\left(\Omega_{\beta}\right)>0$ and $S\left(\Omega_{\beta}\right)<\infty$.

In this section, we give an explicit example, by modifying worm domains, which shows the Diederich-Fornæss and Steinness indices do not admit upper semi-continuity and lower semi-continuity in general, respectively. We first recall the $\beta$-worm domain.

Definition 4.1. The $\beta$-worm domain $D_{\beta}\left(\beta>\frac{\pi}{2}\right)$ is defined by

$$
D_{\beta}:=\left\{(z, w) \in \mathbb{C}^{2}:\left|z-e^{i \log |w|^{2}}\right|^{2}-\left(1-\phi_{\beta}\left(\log |w|^{2}\right)\right)<0\right\}
$$

where $\phi_{\beta}: \mathbb{R} \rightarrow \mathbb{R}$ is a fixed smooth function with the following properties :

(i) $\phi_{\beta}(x) \geq 0, \phi_{\beta}$ is even and convex.

(ii) $\phi_{\beta}^{-1}(0)=I_{\beta-\frac{\pi}{2}}=\left[-\left(\beta-\frac{\pi}{2}\right), \beta-\frac{\pi}{2}\right]$.

(iii) $\exists a>0$ such that $\phi_{\beta}(x)>1$ if $x<-a$ or $x>a$.

(iv) $\phi_{\beta}^{\prime}(x) \neq 0$ if $\phi_{\beta}(x)=1$.

Let $\Omega$ be a domain in $\mathbb{C}^{3}$ defined by the defining function

$$
\rho(z, w, \gamma):=\left|z-e^{i \log |w|^{2}}\right|^{2}-\left(1-\phi_{\frac{3}{4} \pi}\left(\log |w|^{2}\right)-|\gamma|^{2}\right),
$$

i.e., $\Omega:=\left\{(z, w, \gamma) \in \mathbb{C}^{3}: \rho(z, w, \gamma)<0\right\}$, and $\Omega_{\gamma}:=\left\{(z, w) \in \mathbb{C}^{2}: \rho_{\gamma}(z, w):=\right.$ $\rho(z, w, \gamma)<0\}$ for each $\gamma \in \mathbb{D}$. Here, the choice of $\beta=\frac{3}{4} \pi$ is not important in our example.

Now, for the following argument, we refer readers to [6] and [7]. Let

$$
\widetilde{\rho}_{\gamma}(z, w):=\rho_{\gamma}(z, w) e^{2 \arg w}
$$

which is a local defining function near any boundary point $p \in \partial \Omega_{\gamma}$. Note that the holomorphic tangent plain $T_{p}^{1,0}\left(\partial \Omega_{\gamma}\right)$ is spanned by a vector

Then

$$
L_{p}:=-\left.\frac{\partial \rho_{\gamma}}{\partial w}(p) \frac{\partial}{\partial z}\right|_{p}+\left.\frac{\partial \rho_{\gamma}}{\partial z}(p) \frac{\partial}{\partial w}\right|_{p} .
$$

$$
\begin{aligned}
\mathscr{L}_{\widetilde{\rho}_{\gamma}}\left(L_{p}, L_{p}\right)=\frac{e^{2 \arg w}}{4|w|^{2}} & {\left[\left|i \bar{z}\left(z-|w|^{2 i}\right)+\phi_{\frac{3}{4} \pi}^{\prime}\left(\log |w|^{2}\right)\right|^{2}\right.} \\
& \left.+\left.\left.|z-| w\right|^{2 i}\right|^{2}\left(\phi_{\frac{3}{4} \pi}\left(\log |w|^{2}\right)+\phi_{\frac{3}{4} \pi}^{\prime \prime}\left(\log |w|^{2}\right)\right)\right] .
\end{aligned}
$$

Observe that $\left.|z-| w\right|^{2 i} \mid$ never vanish on $\partial \Omega_{\gamma}$. If $\log |w|^{2} \notin\left[-\left(\beta-\frac{\pi}{2}\right), \beta-\frac{\pi}{2}\right]$, then $\phi_{\frac{3}{4} \pi}\left(\log |w|^{2}\right)+\phi_{\frac{3}{4} \pi}^{\prime \prime}\left(\log |w|^{2}\right)>0$ by the definition of $\phi_{\frac{3}{4} \pi}$. If $\log |w|^{2} \in[-(\beta-$ 
$\left.\left.\frac{\pi}{2}\right), \beta-\frac{\pi}{2}\right]$, then $\left|i \bar{z}\left(z-|w|^{2 i}\right)\right|>0$ provided that $z \neq 0$. Therefore, if $\gamma \neq 0$ then $z(p) \neq 0$, hence, $\mathscr{L}_{\widetilde{\rho}_{\gamma}}(L, L)(p)>0$ for all $p \in \partial \Omega_{\gamma}$. This means that $\Omega_{\gamma}$ is strongly pseudoconvex for all $\gamma \neq 0 \in \mathbb{D}$. Since every strongly pseudoconvex domain admits a strictly plurisubharmonic defining function, $D F\left(\Omega_{\gamma}\right)=1$ and $S\left(\Omega_{\gamma}\right)=1$ for all $\gamma \neq 0 \in \mathbb{D}$. Moreover, $\Omega_{0}=D_{\frac{3}{4} \pi}$ implies that $D F\left(\Omega_{0}\right)=\frac{2}{3}$ and $S\left(\Omega_{0}\right)=2$ from the results in [8] and [10]. We conclude that $D F\left(\Omega_{\gamma}\right)$ and $S\left(\Omega_{\gamma}\right)$ are not upper semi-continuous and lower semi-continuous at $\gamma=0$, respectively.

\section{REFERENCES}

[1] M. Adachi and J. Yum, Diederich-Forncess and Steinness indices for abstract CR manifolds (2020), Preprint, available at arXiv:2003.01330.

[2] H. P. Boas and E. J. Straube, de Rham cohomology of manifolds containing the points of infinite type, and Sobolev estimates for the $\bar{\partial}$-Neumann problem, J. Geom. Anal. 3 (1993), no. $3,225-235$.

[3] J. P. D'Angelo, Finite type conditions for real hypersurfaces, J. Differential Geometry 14 (1979), no. 1, 59-66 (1980).

[4] , Iterated commutators and derivatives of the Levi form, Complex analysis (University Park, PA., 1986), Lecture Notes in Math., vol. 1268, Springer, Berlin, 1987, pp. 103-110.

[5] K. Diederich and J. E. Fornæss, Pseudoconvex domains: bounded strictly plurisubharmonic exhaustion functions, Invent. Math. 39 (1977), no. 2, 129-141.

[6] _ Pseudoconvex Domains: an example with nontrivial neighborhood, Math. Ann. 225 (1977), no. 3, 275-292.

[7] S. G. Krantz and M. M. Peloso, Analysis and geometry on worm domains, J. Geom. Anal. 18 (2008), no. 2, 478-510.

[8] B. Liu, The Diederich-Forncess index I: For domains of non-trivial index, Adv. Math. 353 (2019), 776-801.

[9] O. Saeki, Topology of Singular Fibers of Differentiable Maps, Lecture notes in Mathematics, Springer, 2004.

[10] J. Yum, On the Steinness Index, J. Geom. Anal. 29 (2019), no. 2, 1583-1607.

[11] _ CR-invariance of the Steinness index (2019), Preprint, available at arXiv: 1908.01214

Department of Mathematics, Pusan National University, 2, Busandaehak-Ro 63beongil, Geumjeong-Gu, Busan 46241, Republic of Korea

E-mail address: youngjun.choi@pusan.ac.kr

Department of Mathematics, Pusan National University, 2, Busandaehak-ro 63beonGil, Geumjeong-Gu, Busan 46241, Republic of Korea

E-mail address: jihun0224@pusan.ac.kr 